\title{
Intraosseous hemangioblastoma of the cervical spine: case report
}

\author{
Zhenteng Li, MD, ${ }^{1}$ Brian Curtis, MD, ${ }^{1}$ Robert Layser, MD, ${ }^{1}$ Santosh Kumar Selvarajan, MD, ${ }^{1}$ \\ James Harrop, MD, ${ }^{2}$ Lawrence C. Kenyon, MD, PhD, ${ }^{3}$ Theodore Parsons, MD, ${ }^{3}$ and Asa Rubin, MD $^{3}$ \\ Departments of ${ }^{1}$ Radiology, ${ }^{2}$ Neurosurgery, and ${ }^{3}$ Pathology, Thomas Jefferson University Hospital, Philadelphia, Pennsylvania
}

A 69-year-old woman presented with bilateral upper-extremity radiculopathy and neck pain after a mechanical fall. Admission CT and MRI of the cervical spine demonstrated a pathological C-4 fracture. Subsequent malignancy workup was negative. A CT-guided biopsy of the lesion showed intraosseous hemangioblastoma. Hemangioblastoma is a highly vascular, slow-growing tumor of the CNS; intraosseous location of this tumor is extremely rare. The authors review the diversity of its presentation and the treatment techniques of this rare tumor in an extremely rare location.

https://thejns.org/doi/abs/10.3171/2017.3.SPINE1622

KEY WORDS intraosseous hemangioblastoma; cervical spine; oncology

$\mathrm{H}$

EMANGIOBLASTOMAS are rare, slow-growing tumors that are most commonly found in the spinal cord, cerebellum, and brainstem. ${ }^{4}$ They can occur sporadically or as a component of von Hippel-Lindau (VHL) disease; $75 \%$ of them are sporadic and typically solitary. ${ }^{15}$ Solitary sporadic hemangioblastomas have a predilection for the cerebellum, whereas VHL-related tumors are more commonly found in the spinal cord. ${ }^{11}$

Nearly all reported cases of hemangioblastomas are in the CNS, and extraneural locations are extremely rare. Specifically, intraosseous hemangioblastomas are rarely seen. There have been only 2 case reports in the literature on this condition over the last 20 years., ${ }^{3,9}$

Due to their location in bone, hemangioblastomas may be a confounding diagnosis to make, particularly in patients with a known primary malignancy. In patients with no known malignancy, the nonspecific imaging findings may prompt a malignancy workup. To our best knowledge, there have been no described cases of intraosseous hemangioblastomas in the cervical vertebrae. In this report, we describe a case of pathological cervical vertebral fracture secondary to a sporadic, intraosseous hemangioblastoma. We believe that the rarity of its location, unique clinical presentation, nonspecific imaging findings, and subse- quent treatment improve our understanding of this rare tumor, the diversity of its presentation, and its management strategy.

\section{Case Report}

History

A 69-year-old woman presented to our institution with a 2-week history of worsening bilateral upper-extremity pain after a mechanical fall. She initially presented to an urgent care center, where she was given a neck brace and discharged home on naproxen and prednisone. However, her pain worsened. On presentation, she described sharp, intractable pain radiating down the lateral aspect of her arms in the C-5 dermatomal distribution with minimal neck motion.

\section{Physical Examination}

She appeared comfortable in a Miami cervical collar. Her cranial nerve examination revealed left tongue deviation, in keeping with her known history of radiation-treated left vagal nerve schwannoma. The remainder of her neurological examination showed intact status, except $4 / 5$ motor strength in bilateral shoulder abduction. 


\section{Neuroimaging}

A cervical spine CT demonstrated an approximately $25 \%$ loss of height of the C-4 vertebral body, which was largely replaced by soft tissue with no visible internal trabeculae (Fig. 1). Subsequent MRI showed abnormal marrow replacement and enhancement throughout the C-4 vertebral body and posterior elements, sparing the spinous process. Additionally, enhancing soft tissues were present in the prevertebral ventral epidural space, and in the bilateral neural and transverse foramina at the C-4 level, resulting in mild central stenosis. Expansile tumor was spread within the pedicles and facets, obliterating the neural foramina. At this time, the primary radiological differential considerations were metastasis and plasmacytoma.

However, metastatic workup was negative. A CTguided biopsy was performed and showed vascular tumor within bone, consistent with intraosseous hemangioblastoma. Given this diagnosis, selective angiograms were performed for preoperative vascular evaluation and tumor embolization (Fig. 2). Feeding arteries were identified from the muscular branches of $\mathrm{V}_{2}$ segments of the vertebral arteries and thyrocervical trunks. Robust feeders were seen from the thyrocervical trunks and were superselectively embolized with Onyx 18.

\section{Operation}

The patient underwent a C-4 corpectomy with C3-5 anterior and posterior instrumented fusion. Intraoperatively, the longus colli muscles at the $\mathrm{C}-4$ level were infiltrated and hypervascular. The $\mathrm{C}-4$ vertebral body was comminuted.

\section{Pathological Findings}

Macroscopically, a CT-guided core biopsy of the C-4 vertebral body showed 2 fragments of red-pink rubbery soft tissues. Microscopic analysis of the specimens demonstrated that the lesion was within bone and was vasoformative, with a dual population of cells (Fig. 3). Specimen staining with inhibin was noncontributory. Staining with CD56 and neuron-specific enolase (NSE) was positive in foamy cells. Staining with erythroblast transformationspecific-related gene (ERG), CD31, and CD34 was posi- tive in endothelial cells. Staining with MelanA, CD10, AE1/AE3, Cam 5.2, epithelial membrane antigen (EMA), and human herpes virus (HHV)-8 was negative. The Ki-67 level was low. Immunohistochemical profile confirmed the vascular nature of the lesion and did not support metastatic carcinoma or plasmacytoma. These findings were consistent with intraosseous hemangioblastoma. Pathological analysis of the surgical specimens confirmed WHO Grade I hemangioblastoma within bone. At this time, immunohistochemistry for inhibin was positive, further supporting this diagnosis.

\section{Postoperative Course}

At the 1-year follow-up, CT and MR images of the cervical spine showed the spinal cord decompressed with residual tumor lateral to the construct, which was unchanged from immediate postoperative imaging (Fig. 4). At the 1-year point, the patient had done exceptionally well. She was without neck pain and her upper-extremity pain had completely resolved.

\section{Discussion}

The differential diagnosis of a lytic lesion with bone marrow replacement and diffuse enhancement in a cervical vertebra is broad. ${ }^{16}$ However, given the patient's age, the most common etiologies are metastasis, myeloma, and-rarely-atypical hemangiomas. Atypical hemangiomas are rarely completely lucent on CT scans; they typically have vertical internal trabeculae and no diffusion restriction on diffusion-weighted imaging. ${ }^{8}$

Intracranial hemangioblastomas are described as cystic lesions with solid enhancing intramural nodules. Their MR characteristics are hypo- or isointense on T1-weighted imaging and hyperintense on T2-weighted imaging.,11 Due to high vascularity, these tumors typically demonstrate avid, diffuse enhancement and may show vascular flow voids on unenhanced images.

The prevalence of osseous hemangioblastomas is unknown. There have been only 2 reported cases in the last 20 years. ${ }^{3,9}$ In one example, Cho and colleagues reported a case of sporadic osseous hemangioblastoma in a thoracic
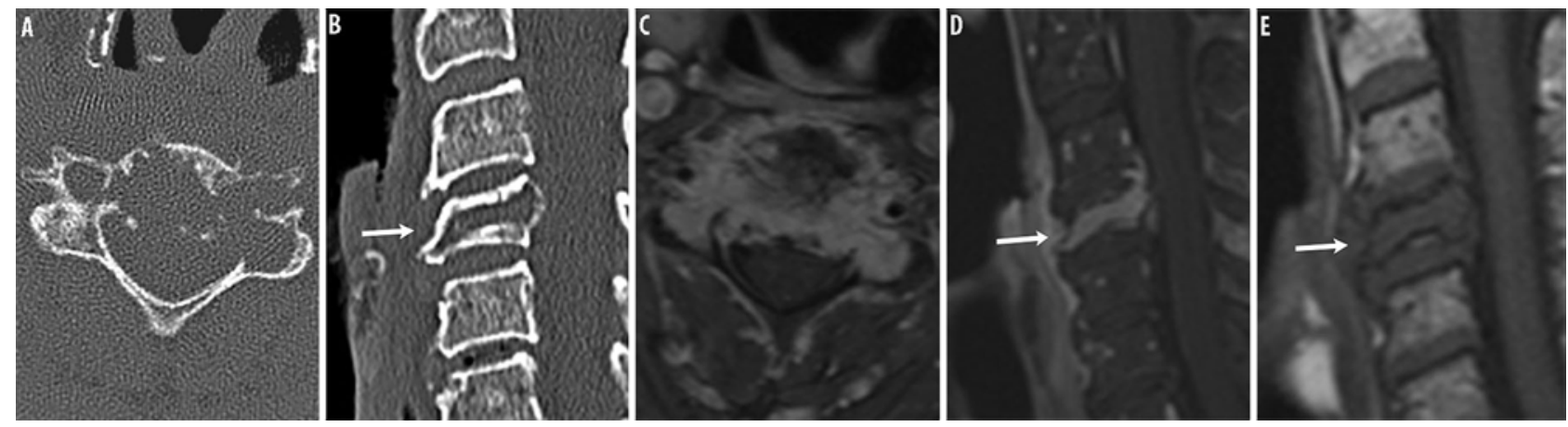

FIG. 1. A C-4 compression fracture secondary to an intravertebral lesion. A and B: Axial and sagittal images showing the C-4 vertebral body largely replaced by irregular, low-density soft tissues with no visible internal trabeculae (arrow). C and D: Axial and sagittal postcontrast fat-suppressed T1-weighted images showing diffuse enhancement throughout the vertebral body, sparing the spinous process. The arrow in panel $D$ designates the $C-4$ vertebral body, which is intensely enhancing on the postcontrast image. E: Sagittal T1-weighted image showing associated marrow replacement. The arrow in panel E designates the C-4 vertebral body, which is homogeneously hypointense, suggesting marrow replacement in the precontrast image. 

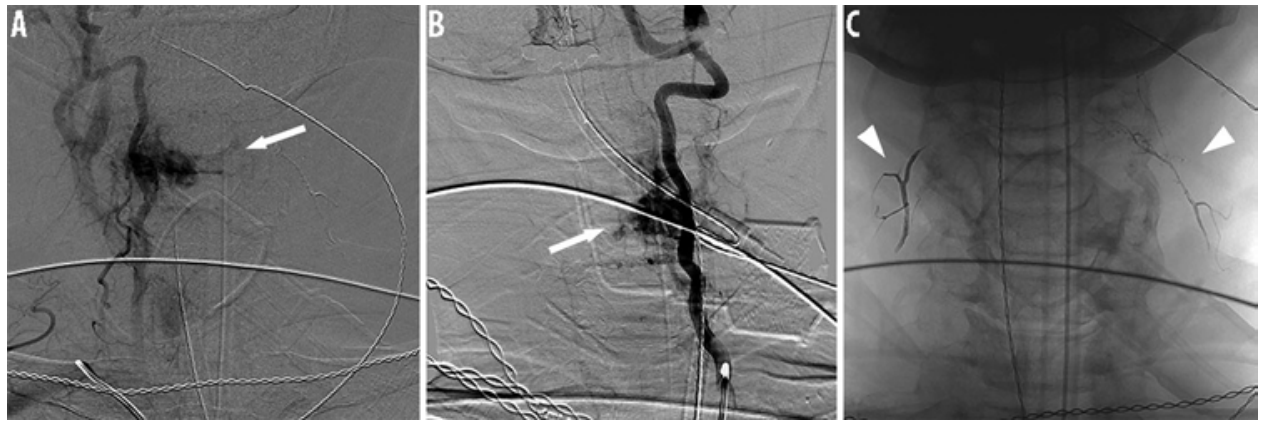

FIG. 2. A and B: Preoperative digital subtraction angiogram showing neovascularity of the C-4 intravertebral mass, with feeding vessels from $\mathrm{V}_{2}$ segments of the vertebral arteries and thyrocervical trunks (arrows). C: Postembolization frontal image showing successful embolization of feeding vessels from the thyrocervical trunks (arrowheads).

vertebra, which was initially suspected to be a metastasis on imaging in a patient with a history of renal cell carcinoma. ${ }^{3}$ Given its unusual location, this type of intraosseous tumor can only be suggested as a diagnosis of exclusion prior to biopsy. Our case demonstrated avid enhancement indicative of a highly vascular tumor. However, this is a rather nonspecific feature of hemangioblastomas. Often, there are overlapping imaging features between this rare tumor and other much more common etiologies, such as metastasis, myeloma, and lymphoma. Even with clinical and imaging findings raising suspicion, the diagnosis can only be made with pathological analysis.

There is no clear guideline for the management of intraosseous hemangioblastomas. Treatments similar to those used for its counterparts within the neuraxis were performed in the reported cases. ${ }^{3}$ Anatomical location of
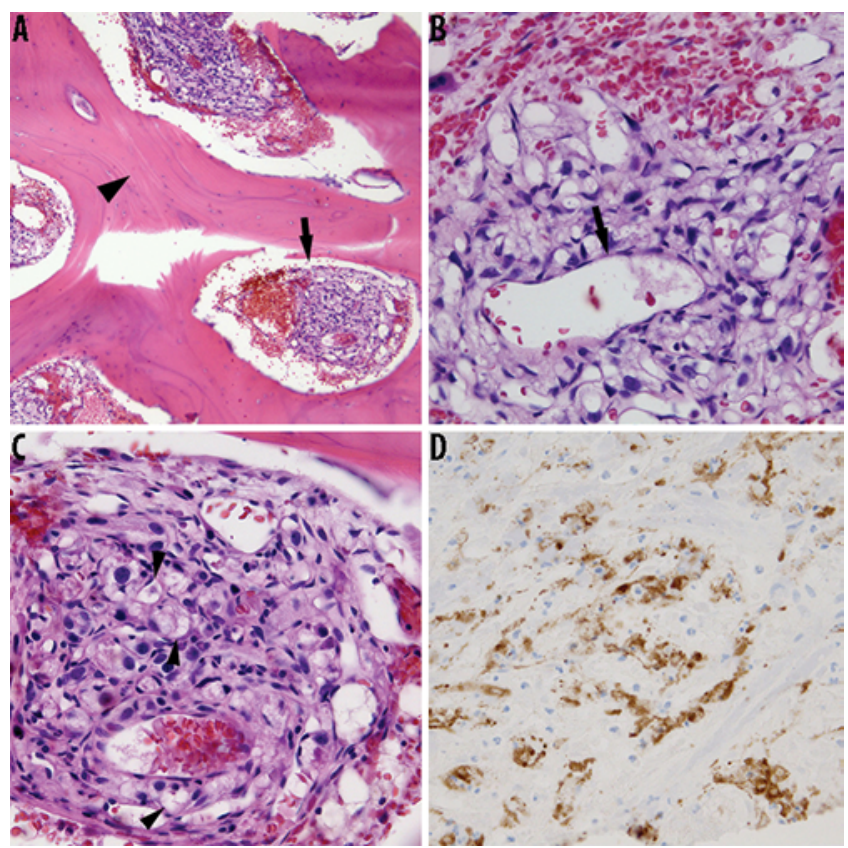

FIG. 3. Photomicrographs of the resected tumor. A: Low-magnification $(\times 100) H$ \& E section showing a vasoformative lesion (arrow) within bone (arrowhead). B and C: High-magnification $(\times 400) \mathrm{H} \& \mathrm{E}$ sections showing endothelial cells (arrow) and stromal cells with foamy cytoplasm (arrowheads). D: Cells stained positive with inhibin. Figure is available in color online only. the tumor is an important factor in determining the therapeutic approach. Small or asymptomatic tumors may be followed with imaging to ensure stability, until they become enlarged or symptomatic..$^{18,19}$ There are 2 treatment modalities available for intraaxial hemangioblastomas: resection and radiation therapy. For solitary tumors in surgically accessible locations, surgical removal often offers definitive treatment with acceptable postoperative morbidities., ${ }^{72,13,19,20}$ Due to the highly vascular nature of these tumors, surgery is typically supplemented by preoperative embolization, which minimizes intraoperative blood loss and allows for complete resection of the tumor using a microdissection technique..$^{1,5,6,17}$ On the other hand, radiation therapy is considered a feasible alternative for
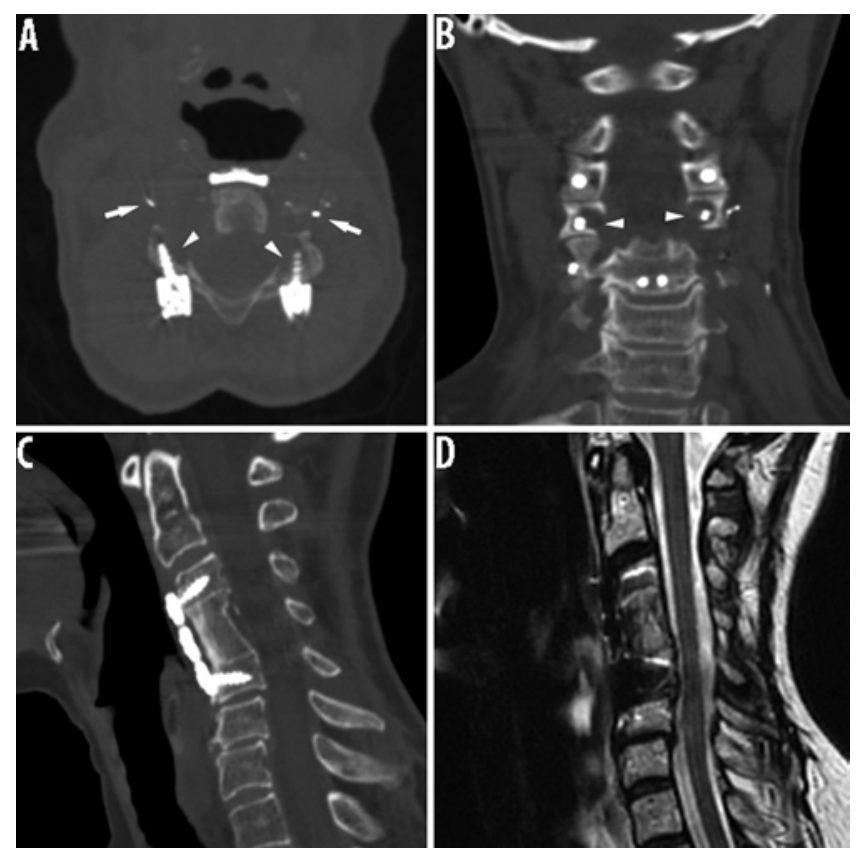

FIG. 4. One-year postoperative imaging. A-C: Axial, coronal, and sagittal images showing a well-incorporated strut graft between $\mathrm{C}-3$ and C-5 and good position of the anterior and posterior fusion construct. Residual tumor is noted in bilateral C-4 lateral masses (arrowheads) and is unchanged compared with the preoperative study. Embolization materials (arrows) are also noted. D: Sagittal T2-weighted image showing capacious central canal without cord compromise. Residual tumor is not well seen on MRI due to susceptibility artifact. 
surgically inaccessible lesions or VHL-related, multifocal lesions. ${ }^{3,10,14}$ Regardless of treatment modality, the primary goal of therapy is to avoid treatment-related morbidities. This patient became symptomatic after pathological fracture of a cervical vertebra secondary to intravertebral hemangioblastoma. Fortunately for her, the hemangioblastoma was extradural and intravertebral in location, which allowed complete resection with no significant complications.

\section{Conclusions}

Intraosseous hemangioblastoma should be considered in the differential diagnosis of isolated intravertebral low-density lesions with marrow replacement and avid enhancement, after common etiologies are excluded. In reporting this case, we illustrate the diversity of presentation of hemangioblastomas and the effective management techniques of this rare tumor in an extremely rare location.

\section{References}

1. Ampie L, Choy W, Khanna R, Smith ZA, Dahdaleh NS, Parsa AT, et al: Role of preoperative embolization for intradural spinal hemangioblastomas. J Clin Neurosci 24:83-87, 2016

2. Baker KB, Moran CJ, Wippold FJ II, Smirniotopoulos JG, Rodriguez FJ, Meyers SP, et al: MR imaging of spinal hemangioblastoma. AJR Am J Roentgenol 174:377-382, 2000

3. Cho H, Lee SH, Kim ES, Eoh W: Intraosseous hemangioblastoma mimicking spinal metastasis in the patient with renal cell carcinoma. J Korean Neurosurg Soc 49:381-383, 2011

4. Conway JE, Chou D, Clatterbuck RE, Brem H, Long DM, Rigamonti D: Hemangioblastomas of the central nervous system in von Hippel-Lindau syndrome and sporadic disease. Neurosurgery 48:55-63, 2001

5. dos Santos MP, Zhang J, Ghinda D, Glikstein R, Agid R, Rodesch G, et al: Imaging diagnosis and the role of endovascular embolization treatment for vascular intraspinal tumors. Neurosurg Focus 39(2):E16, 2015

6. Eskridge JM, McAuliffe W, Harris B, Kim DK, Scott J, Winn HR: Preoperative endovascular embolization of craniospinal hemangioblastomas. AJNR Am J Neuroradiol 17:525-531, 1996

7. Fukuda M, Takao T, Hiraishi T, Yoshimura J, Yajima N, Saito A, et al: Clinical factors predicting outcomes after surgical resection for sporadic cerebellar hemangioblastomas. World Neurosurg 82:815-821, 2014

8. Hatipoglu HG, Selvi A, Ciliz D, Yuksel E: Quantitative and diffusion MR imaging as a new method to assess osteoporosis. AJNR Am J Neuroradiol 28:1934-1937, 2007

9. Higgins JN, Lammie GA, Savy LE, Taylor WJ, Stevens JM: Intraosseous vertebral haemangioblastoma: MRI. Neuroradiology 38 (Suppl 1):S107-S110, 1996

10. Kano H, Niranjan A, Mongia S, Kondziolka D, Flickinger JC, Lunsford LD: The role of stereotactic radiosurgery for intracranial hemangioblastomas. Neurosurgery 63:443-451, 2008
11. Leung RS, Biswas SV, Duncan M, Rankin S: Imaging features of von Hippel-Lindau disease. Radiographics 28:6579, 323, 2008

12. Liu A, Jain A, Sankey EW, Jallo GI, Bettegowda C: Sporadic intramedullary hemangioblastoma of the spine: a single institutional review of 21 cases. Neurol Res 38:205-209, 2016

13. Lonser RR, Weil RJ, Wanebo JE, DeVroom HL, Oldfield EH: Surgical management of spinal cord hemangioblastomas in patients with von Hippel-Lindau disease. J Neurosurg 98:106-116, 2003

14. Moss JM, Choi CY, Adler JR Jr, Soltys SG, Gibbs IC, Chang SD: Stereotactic radiosurgical treatment of cranial and spinal hemangioblastomas. Neurosurgery 65:79-85, 2009

15. Neumann HP, Eggert HR, Weigel K, Friedburg H, Wiestler $\mathrm{OD}$, Schollmeyer P: Hemangioblastomas of the central nervous system. A 10-year study with special reference to von Hippel-Lindau syndrome. J Neurosurg 70:24-30, 1989

16. Rodallec MH, Feydy A, Larousserie F, Anract P, Campagna R, Babinet A, et al: Diagnostic imaging of solitary tumors of the spine: what to do and say. Radiographics 28:1019-1041, 2008

17. Tampieri D, Leblanc R, TerBrugge K: Preoperative embolization of brain and spinal hemangioblastomas. Neurosurgery 33:502-505, 1993

18. Wanebo JE, Lonser RR, Glenn GM, Oldfield EH: The natural history of hemangioblastomas of the central nervous system in patients with von Hippel-Lindau disease. J Neurosurg 98:82-94, 2003

19. Weil RJ, Lonser RR, DeVroom HL, Wanebo JE, Oldfield EH: Surgical management of brainstem hemangioblastomas in patients with von Hippel-Lindau disease. J Neurosurg 98:95-105, 2003

20. Westwick HJ, Giguère JF, Shamji MF: Incidence and prognosis of spinal hemangioblastoma: a Surveillance Epidemiology and End Results study. Neuroepidemiology 46:14-23, 2016

\section{Disclosures}

The authors report no conflict of interest concerning the materials or methods used in this study or the findings specified in this paper.

\section{Author Contributions}

Conception and design: Li. Acquisition of data: Li, Curtis, Layser, Harrop, Kenyon, Parsons. Analysis and interpretation of data: Selvarajan, Li, Curtis, Layser, Harrop, Kenyon. Drafting the article: Li. Critically revising the article: Selvarajan, Li, Curtis, Layser, Harrop. Reviewed submitted version of manuscript: Selvarajan, Li, Curtis, Layser, Harrop, Kenyon, Parsons. Approved the final version of the manuscript on behalf of all authors: Selvarajan. Administrative/technical/material support: Rubin. Study supervision: Selvarajan, Li.

\section{Correspondence}

Santosh Kumar Selvarajan, TJUH Department of Radiology, 132 South 10th St., Ste. 1087, Main Bldg., Philadelphia, PA 19107. email: santosh.selvarajan@jefferson.edu. 